\title{
Boundedness of fractional differential operator in complex spaces
}

\begin{abstract}
In this work, we introduce some properties of a complex fractional differential operator. The boundedness and some other properties are studied in view of geometric function theory.

Keyword: Unit disk; Analytic functions; Univalent function; Fractional differential operator; Convolution product; Laguerre functions; Hypergeometric function
\end{abstract}

